\title{
Sensing single molecule under micromanipulation to quantify nucleic acids
}

\section{Qiang Zeng}

Shanghai Jiaotong University https://orcid.org/0000-0002-2759-422X

Xiaoyan Zhou

Shanghai Jiaotong University

\section{Yuting Yang}

Shanghai Jiao Tong University

Jingan Wang

Shanghai Jiao Tong University

Chunhui Zhai

Shanghai Jiao Tong University

Hui Yu ( $\square$ hui.yu@sjtu.edu.cn )

Shanghai Jiao Tong University https://orcid.org/0000-0003-4430-2230

\section{Brief Communication}

Keywords: analytical chemistry, molecuplar diagnosis, biomarkers, mRNAs

Posted Date: June 14th, 2021

DOl: https://doi.org/10.21203/rs.3.rs-598436/v1

License: (c) (i) This work is licensed under a Creative Commons Attribution 4.0 International License.

Read Full License 


\section{Sensing single molecule under micromanipulation to quantify nucleic acids}

Qiang Zeng ${ }^{1 \#}$, Xiaoyan Zhou ${ }^{1 \#}$, Yuting Yang ${ }^{2 \#}$, Jingan Wang ${ }^{1}$, Chunhui Zhai ${ }^{1}$, Hui Yu $^{1,3^{*}}$

${ }^{1}$ School of Biomedical Engineering, Shanghai Jiao Tong University, Shanghai, 200030,

People's Republic of China

${ }^{2}$ Department of Instrument Science and Engineering, School of Electronic Information and Electrical Engineering, Shanghai Jiao Tong University, Shanghai, 200030, People's Republic of China

${ }^{3}$ Institute of Medical Robotics, Shanghai Jiao Tong University, Shanghai, 200030, People's Republic of China

${ }^{\#}$ These authors contributed equally to this work.

*Correspondence should be addressed to: H.Y. (hui.yu@sjtu.edu.cn) 
Abstract

Rapid molecular diagnosis using nucleic acid biomarkers is important for timely identification of acute pathogenic infections. We introduce an active kinetic approach called sensing single molecule under micromanipulation $\left(\mathrm{SSM}^{3}\right)$ to quantify nucleic acids, which circumvents hydrodynamic limits of reaction rate, sensitivity and specificity. We demonstrate the 15-minute assay to detect synthetic miRNAs with subfemtomolar limit of detection and high-confidence discrimination of single-base-pair mismatch. 
Nucleic acid biomarkers from various origins have been widely used in disease diagnosis ${ }^{1}$, but timely quantification of nucleic acids with sufficient sensitivity has been challenging. The standard assays in clinical settings are based on polymerase chain reaction (PCR), which usually requires time-consuming extraction and amplification steps. ${ }^{2}$ For example, the practical nucleic acid tests of coronavirus disease (COVID-19) take hours ${ }^{3}$, which could delay the prevention of spread. Several amplification-free approaches have been developed by digitally counting single nucleic acid molecules in either conventional endpoint assays ${ }^{4,5}$ or more advanced kinetic assays. $^{6-9}$ Especially, state-of-the-art single-molecule recognition through equilibrium Poisson sampling (SiMREPS) have achieved ultrahigh sensitivity and specificity by exploiting the repeated binding of a short fluorescently labeled DNA probe to an analyte immobilized on sensor surfaces. ${ }^{6,10,11}$ However, these typically require incubation of analyte molecules onto the sensor surface for at least tens of minutes before detection, due to the limitation by mass transportation and thermodynamics of hybridization. ${ }^{6,7,12,13}$ (Supplementary Note 1)

Here we present bioconjugated nanoparticles assisted single molecule kinetic assay to circumvent the above limits in nucleic acid detection. In the sensing single molecule under micromanipulation $\left(\mathrm{SSM}^{3}\right.$ ) approach (Fig. 1a), surface plasmon resonance microscopy (SPRM) ${ }^{14}$ dynamically images DNA probe conjugated nanoparticles as the reporter of binding and dissociation events (Fig. 1b). To beat the diffusion limit, analyte molecules are captured by longer detection probes (12- nucleotides, nt) conjugated to the nanoparticles and actively brought to the sensor surface by applying an external driving force (Supplementary Fig.1). The use of longer probes ensures a higher binding rate to capture analyte, but it also inevitably slows down the dissociation rate. Inspired by the single molecule force spectroscopy ${ }^{15,16}$, in $\mathrm{SSM}^{3}$, we tuned the 
dissociation rate by applying a load on the molecular bound through micromanipulation of the nanoparticles to promote the repeated binding (Supplementary Fig.1 and Note 2). In our customized SPRM system, each binding event is discriminated to be specific molecular binding or nonspecific background binding by analyzing the bound lifetime related to the distinctive kinetic signature (Supplementary Fig.2).

a

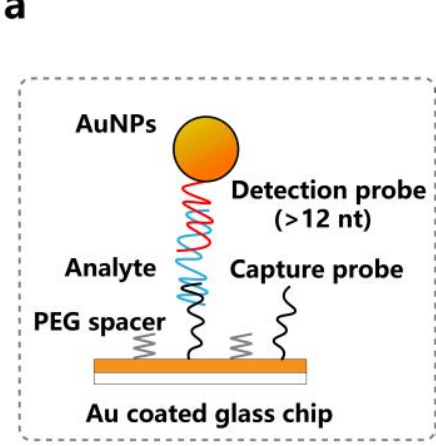

C

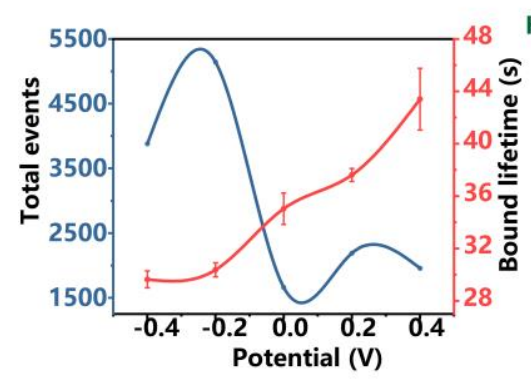

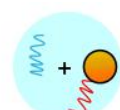
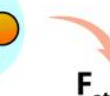

$F_{\text {attraction }}$
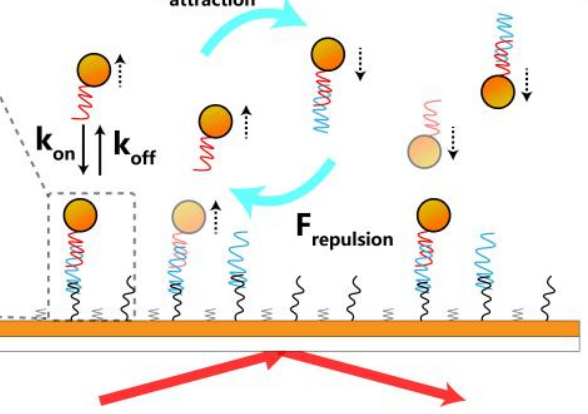

d

Hit and run Specific binding Hit and absorb b

$\square$ binding $\square$ dissociation

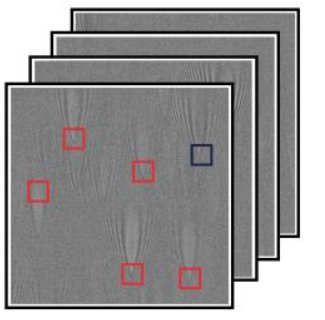

e
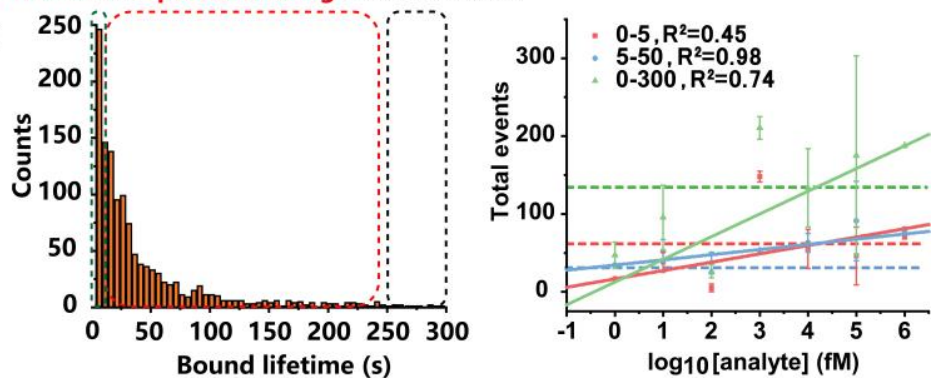

Fig.1 Detection of nucleic acids with $\mathrm{SSM}^{3}$. (a) Schematic illustration of $\mathrm{SSM}^{3}$. (b) Image acquisition and processing. (c) The tuning of bound lifetime and number of binding and dissociation events with different potential applied. (d) Setting time windows to identify specific bindings of $h s a-m i R-29 a$. (e) Sensing performance of $h s a-m i R-29 a$ when selecting different time windows for counting binding events including 0-5 s, 5-50 s and 0-300 s in (d). Dashed lines are the control without analyte.

As an example, we explored the micromanipulation of DNA probes conjugated on 50-nm Au nanoparticles (AuNPs) by applying an electrical field between the Au surface and a reference 
electrode in solution, owing to the compatibility of SPRM with electrochemical systems. ${ }^{17,18}$ The number of total binding and dissociation events $(N)$ and the bound lifetime $\left(t_{b}\right)$ for a probe with 12-nt complementary bases (Supplementary Table 1) were analyzed when different voltages were applied (Fig. 1c). Consistent with our expectation, $t_{\mathrm{b}}$ decreased with a pulse force at negative voltages, indicating the tuning of dissociation rate. But $N$ increased by over 3 folds at $0.2 \mathrm{~V}$ (Supplementary Note 3). With the micromanipulation, the specific bindings to microRNA (miRNA) hsa-miR-29a (miR-29a) could be identified by applying an appropriate window on the $t_{\mathrm{b}}$ histogram to reject nonspecific bindings (Fig.1d). When choosing the 5-50 s window, the sensitivity and linearity in concentration detection were greatly improved (Fig. 1e).

While longer detection time ensures higher sensitivity and specificity (Supplementary Fig. 3), we demonstrated a 15-minute assay to prove the effectiveness and generality using three miRNAs including hsa-miR-21-5p (miR-21), hsa-miR-155-5p (miR-155) and hsa-miR-362-5p (miR-362) (Fig.2a), which is acceptable waiting time in an out-patient visit. Despite varied binding kinetics, $t_{\mathrm{b}}$ were all separated from unspecific bindings by setting a threshold at $5 \mathrm{~s}$ (Supplementary Fig. 4). Digital counting of only specific binding events provides ultrahigh sensitivity with subfemtomolar limit of detection (LoD) (Fig. 2b). The LoD for miR-21, miR-155 and $m i R-362$ were $0.26,0.17$ and $0.15 \mathrm{fM}$ respectively, with linear detection ranges over 7 orders of magnitude. In the receiver operating characteristic (ROC) plot, the area under curve (AUC) values were 0.997 for $m i R-21,0.988$ for $m i R-155$ and 0.967 for $m i R-362$, indicating high specificity against nonspecific bindings (Fig. 2c). A more rigorous threshold setting could further improve the specificity, but at the cost of less countable events. 


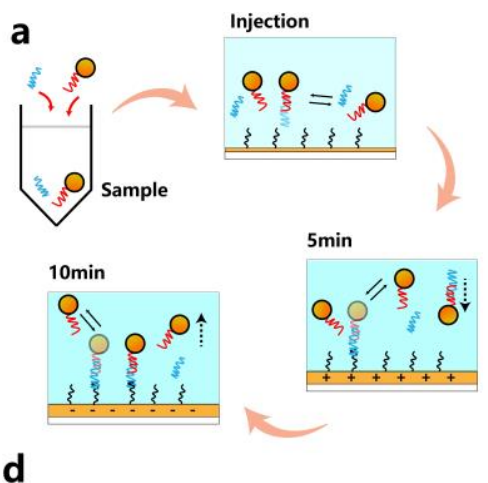

b

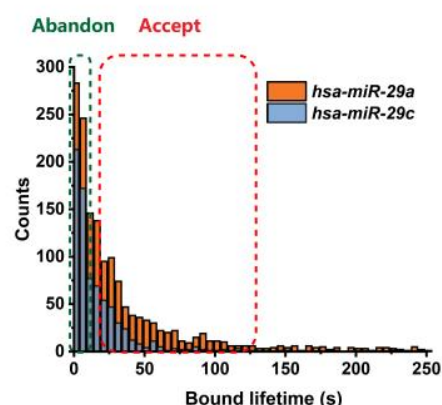

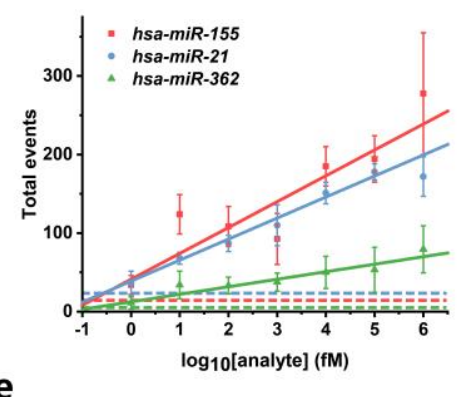

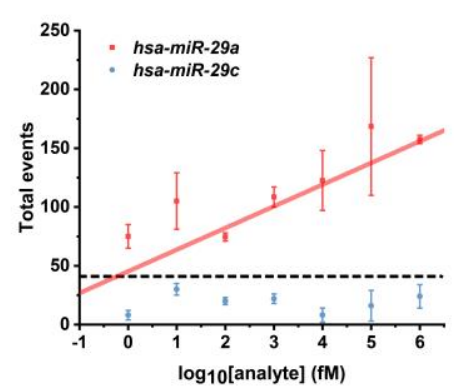

C

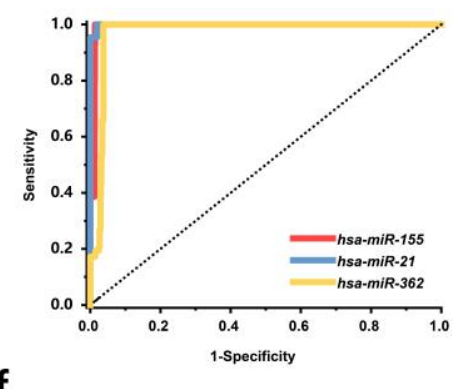

f

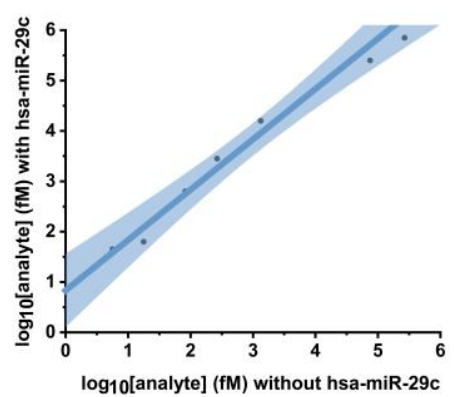

Fig.2 The 15-minute assay for miRNA detection and single nucleotide mismatch discrimination.

(a) Workflow of the 15-minute assay. (b) Calibration curves and corresponding (c) ROC curves of three disease-related miRNAs. Dashed lines represent the reference without analyte (mean $+3 \times$ std). (d) Bound time filter for high-confidence distinguishing hsa-miR-29a from hsa-miR-29c. (e) Calibration curves of hsa-miR-29a and hsa-miR-29c. The dashed line indicates the number of nonspecific binding events. (f) The detection of $h s a-m i R-29 a$ with or without the presence of 100-fold higher concentration of hsa-miR-29c. Blue region indicates the $95 \%$ confidence level. All experiments were performed in triplicates.

The 15-minute SSM ${ }^{3}$ assay could also discriminate single-base mismatch. Two diseases-related miRNAs, the miR-29a and hsa-miR-29c (miR-29c), were synthesized with a single nucleotide mismatch. The $t_{\mathrm{b}}$ were found to be $21.47 \pm 0.45 \mathrm{~s}$ and $10.42 \pm 0.96 \mathrm{~s}$ (mean $\pm \mathrm{std}$ ) respectively with an exponential fitting (Fig. 2d). We thus empirically set a threshold at $14 \mathrm{~s}$, and observed the distinct sensing performance for miR-29a and miR-29c with a discrimination factor over 141 (Fig. 2e and Supplementary Note 4). Even in the presence of miR-29c at a 100 -fold higher concentration, the LoD of miR-29a was $0.63 \mathrm{fM}$ with an AUC of 0.816 (Supplementary Fig. 4). The 
detection of miR-29a with or without the existence of miR-29c further verified the effectiveness (slope $=0.92, \mathrm{R}>0.99)($ Fig.2f).

We have presented a method for rapid detection of specific nucleic acid biomarkers by fine tuning of molecular binding kinetics through nanoparticle micromanipulation. The $\mathrm{SSM}^{3}$ enables the 15-minute assay with subfemtomolar detection limit and single base mismatch discrimination, which is a critical step towards practical testing in an outpatient visit.

\section{METHODS}

Methods and any associated references are submitted separately.

\section{ACKNOWLEDGMENTS}

This work was funded by the National Major Scientific Research Instrument Development Program (Grant 22027807) and the National Natural Science Foundation of China (Grants 61901257 and 61805136) for financial support. The authors thank Dr. Hongchen Gu and Dr. Hong Xu for helpful discussions.

\section{AUTHOR CONTRIBUTIONS}

H.Y. conceived the idea. Q.Z. Y.Y. and H.Y. designed the experiments. Q.Z., X.Z. and Y.Y. carried out the experiments and analyzed the results. Q.Z., X.Z., Y.Y. J.W., C.Z. and H.Y. interpreted the results and wrote the paper.

\section{COMPETING FINANCIAL INTERESTS}

The authors declare no competing interests.

\section{REFERENCES}

1. Schwarzenbach, H., Hoon, D.S. \& Pantel, K. Nat. Rev. Cancer 11, 426-437 (2011).

2. White, A.K. et al. Proc. Natl. Acad. Sci. USA 108, 13999-14004 (2011).

3. Aman, R., Mahas, A. \& Mahfouz, M. ACS Synth. Biol. 9, 1226-1233 (2020).

4. $\quad \mathrm{Xu}, \mathrm{W}$. , Yin, P. \& Dai, M. Angew. Chem. Intl. Ed. 130, 14271-14275 (2018).

5. Cohen, L., Hartman, M.R., Amardey-Wellington, A. \& Walt, D.R. Nucleic Acids Res. 45, e137-e137 (2017).

6. Johnson-Buck, A. et al. Nat. Biotechnol. 33, 730-732 (2015).

7. Sevenler, D., Trueb, J. \& Ünlü, M.S. Proc. Natl. Acad. Sci. USA 116, 4129-4134 (2019).

8. Visser, E.W., Yan, J., van IJzendoorn, L.J. \& Prins, M.W. Nat. Commun. 9, 1-10 (2018).

9. Canady, T.D. et al. Proc. Natl. Acad. Sci. USA 116, 19362-19367 (2019).

10. Johnson-Buck, A., Li, J., Tewari, M. \& Walter, N.G. Methods 153, 3-12 (2019).

11. Hayward, S.L. et al. J. Am. Chem. Soc. 140, 11755-11762 (2018).

12. Squires, T.M., Messinger, R.J. \& Manalis, S.R. Nat. Biotechnol. 26, 417-426 (2008). 
13. Zhang, D.Y., Chen, S.X. \& Yin, P. Nat. Chem. 4, 208-214 (2012).

14. Yang, Y. et al. Proc. Natl. Acad. Sci. USA 115, 10275-10280 (2018).

15. Merkel, R., Nassoy, P., Leung, A., Ritchie, K. \& Evans, E. Nature 397, 50-53 (1999).

16. Strunz, T., Oroszlan, K., Schäfer, R. \& Güntherodt, H.-J. Proc. Natl. Acad. Sci. USA 96, 11277-11282 (1999).

17. Shan, X. et al. Nat. Nanotechnol. 7, 668 (2012).

18. Shan, X., Patel, U., Wang, S., Iglesias, R. \& Tao, N. Science 327, 1363-1366 (2010). 


\section{Supplementary Files}

This is a list of supplementary files associated with this preprint. Click to download.

- Slready.pdf 